\title{
Drift waves in stellarator geometry
}

\author{
M Persson $\dagger$, M Nadeem $\dagger$, J L V Lewandowski $\ddagger$ and H J Gardner $\ddagger \|$ \\ $\dagger$ Department of Electromagnetics, Chalmers University of Technology, S-41296 Göteborg, \\ Sweden \\ \$ Plasma Research Laboratory and Department of Theoretical Physics, \\ Research School of Physical Sciences and Engineering, The Australian National University, \\ Canberra, ACT 0200, Australia
}

Received 8 June 1999, in final form 16 September 1999

\begin{abstract}
Drift waves are investigated in a real three-dimensional stellarator geometry. A linear system, based on the cold ion fluid model and a ballooning mode formalism, is solved numerically in the geometry of the stellarator H1-NF. The spectra of stable and unstable modes, as well as localization, are discussed. The dependence of the spectrum of the unstable modes on the wavevector, plasma density variation, and the location in the plasma is presented.
\end{abstract}

\section{Introduction}

Drift wave instabilities are considered as a strong candidate to explain the large cross-field anomalous energy transport in plasma fusion devices (Tang et al 1986, Boozer et al 1990, Stroth 1998, Redd et al 1999). They have therefore been intensively investigated over the last few decades (Rutherford and Frieman 1968, Pearlstein and Berk 1969, Ross and Mahajan 1978, Hastie et al 1979, Strand et al 1998). However, most studies are carried out using simplified magnetic field configurations. This is true for stellarators although, with their threedimensional geometry, they lend themselves poorly to such treatment.

In this paper we investigate drift instabilities in three-dimensional stellarator geometry using the VMEC code (Hirshman and Betancourt 1991) to generate the equilibria. In particular, we focus on the H1-NF heliac (Hamberger et al 1990). In doing so we extend earlier work (Bhattacharjee et al 1983, Persson et al 1996, Persson and Lewandowski 1997) using helically symmetric models and complement the work on the ATF stellarator (Dominguez et al 1992). We have developed a computer code that calculates the necessary quantities in Boozer coordinates from VMEC output and solves an eigenvalue problem for the cold ion drift wave. In the absence of an explicit driving mechanism, wide spectra of highly stable and marginally stable eigenmodes are found around every selected point in the plasma. The former have low frequencies and are extended along the field lines. The latter have larger frequencies and are localized along the field line. The localized modes can be driven unstable through dissipative or resonance mechanisms. The existence of unstable localized modes then depends on the orientation of the perpendicular wavevector and the plasma density variation, with the highly localized modes being excited only around the symmetry points of the magnetic field configuration.

$\S$ Present address: Princeton Plasma Physics Laboratory (Theory Division), Princeton, NJ 08543, USA

\| Also at: Faculty of Engineering and Information Technology.

0741-3335/99/020203+14\$19.50 @ 1999 IOP Publishing Ltd 
This paper is organized as follows: in section 2 we explain the magnetic field configuration and the coordinate systems used; the drift wave model is discussed in section 3 ; the numerical results are presented in section 4 ; and the paper is concluded with a discussion of the results in section 5 .

\section{Magnetic field configuration}

After a coordinate transformation from the VMEC coordinate system the equilibrium field configurations are expressed in terms of Boozer coordinates $(s, \theta, \zeta)$ (D'haeseleer et al 1991), where $\theta$ and $\zeta$ are generalized poloidal and toroidal angles and $s=2 \pi \psi / \psi_{\mathrm{p}}$ is the normalized flux (radial) coordinate. Here $2 \pi \psi$ is the poloidal magnetic flux bounded by the magnetic axis and the $\psi=$ const surface, and $\psi_{\mathrm{p}}=\pi B_{\mathrm{o}} \bar{a}^{2} / q$ is the total poloidal magnetic flux, where $B_{\mathrm{o}}$ is the magnetic field at the magnetic axis, $\bar{a}$ is the average minor radius, $q=2 \pi / \iota$ is the safety factor and $\iota=\iota(\psi)$ is the rotational transform.

In this coordinate system the magnetic field $\boldsymbol{B}$ is expressed as

$$
\boldsymbol{B}=\nabla \alpha \times \nabla \psi=\dot{\psi} \nabla \alpha \times \nabla s \quad \text { with } \dot{\psi} \equiv \frac{\mathrm{d} \psi}{\mathrm{d} s}=\frac{B_{\mathrm{o}} \bar{a}^{2}}{2 q} \text { and } \alpha=\zeta-q \theta .
$$

This fulfills $\nabla \cdot \boldsymbol{B}=0$ and $\boldsymbol{B} \cdot \nabla \psi=\boldsymbol{B} \cdot \nabla \alpha=0$, which implies that $\psi$ and $\alpha$ are stream functions of the magnetic field. It further implies that $\psi=$ const is a magnetic flux surface and $\alpha=$ const is a field line label on this surface. Topologically, the magnetic flux surface is a square cell, $0 \leqslant \theta<2 \pi, 0 \leqslant \zeta<2 \pi$, in which the field lines are straight. For the eigenvalue problem, along the field line discussed in the next section, complete straight magnetic field lines lie in the topological space $-\infty<\theta<\infty,-\infty<\zeta<\infty$, which is usually referred to as a covering space (Dewar and Glasser 1983).

A Cartesian coordinate system $(x, y, z)$ is related to the Boozer system through the cylindrical coordinates $(R, \phi, z)$, where $R$ is the major axis and $\phi$ the cylindrical toroidal angle as

$$
\begin{aligned}
& R=\sum_{m=0}^{n_{\mathrm{p}}} \sum_{n=-n_{\mathrm{t}}}^{n_{\mathrm{t}}} R_{m n}(s) \cos (m \theta+n N \zeta) \\
& \phi=\zeta-\frac{2 \pi}{N} \sum_{m=0}^{n_{\mathrm{p}}} \sum_{n=-n_{\mathrm{t}}}^{n_{\mathrm{t}}} \phi_{m n}(s) \sin (m \theta+n N \zeta) \\
& z=\sum_{m=0}^{n_{\mathrm{p}}} \sum_{n=-n_{\mathrm{t}}}^{n_{\mathrm{t}}} z_{m n}(s) \sin (m \theta+n N \zeta)
\end{aligned}
$$

Here $n_{\mathrm{p}}$ and $n_{\mathrm{t}}$ are the maximum poloidal and toroidal Fourier components, respectively, and $N$ is the number of field periods of the stellarator studied. The Fourier coefficients $R_{m n}, \phi_{m n}$ and $z_{m n}$, and the rotational transform $\iota$ are calculated as functions of the flux coordinate $s$ by the VMEC code (Hirshman and Betancourt 1991) and then mapped over to the Boozer coordinate system.

The position vector $r$ of any point $(s, \theta, \zeta)$ at a magnetic surface $s$ in the Cartesian coordinate system is

$$
r=(R \cos \phi, R \sin \phi, z)
$$

with the covariant basis vectors of the Boozer coordinate system

$$
\boldsymbol{e}_{s}=\frac{\partial \boldsymbol{r}}{\partial s} \quad \boldsymbol{e}_{\theta}=\frac{\partial \boldsymbol{r}}{\partial \theta} \quad \boldsymbol{e}_{\zeta}=\frac{\partial \boldsymbol{r}}{\partial \zeta}
$$


and the associated contravariant basis vectors

$$
\nabla s=\frac{e_{\theta} \times e_{\zeta}}{J} \quad \nabla \theta=\frac{e_{\zeta} \times e_{s}}{J} \quad \nabla \zeta=\frac{e_{s} \times e_{\theta}}{J} .
$$

Here, the Jacobian $J$ for the Boozer coordinates system is given by

$$
J \equiv e_{s} \cdot e_{\theta} \times e_{\zeta}=\frac{\bar{R} \dot{\psi}}{B^{2}}\left(B_{\theta}+q B_{\zeta}\right)
$$

where $B_{\theta}$ and $B_{\zeta}$, the covariant components of $\boldsymbol{B}$, are surface quantities, i.e. $B_{\theta}=B_{\theta}(s)$ and $B_{\zeta}=B_{\zeta}(s)$. Thus, having calculated numerically the derivatives of the cylindrical coordinates $r, \phi$, and $z$ w.r.t. $s$, equation (2) can be used to calculate derivatives of $B$ w.r.t. $\theta$ and $\zeta$ analytically, while the derivative w.r.t. $s$ has to be calculated numerically.

By using the fact that the contravariant and covariant basis vectors are reciprocal of each other, equation (1) can be written as

$$
\boldsymbol{B}=\frac{\dot{\psi}}{J}\left(\boldsymbol{e}_{\theta}+q \boldsymbol{e}_{\zeta}\right)
$$

So, the operator $e_{\|} \cdot \nabla$, where $e_{\|}=B / B$, is

$$
e_{\|} \cdot \nabla=\frac{\dot{\psi}}{J B}\left(\frac{\partial}{\partial \theta}+q \frac{\partial}{\partial \zeta}\right)=\left.\frac{\dot{\psi} q}{J B} \frac{\mathrm{d}}{\mathrm{d} \zeta}\right|_{\text {field line }}
$$

and the field line curvature vector $\kappa$ is

$$
\boldsymbol{\kappa} \equiv \boldsymbol{e}_{\|} \cdot \nabla \boldsymbol{e}_{\|}=q\left(\frac{\dot{\psi}}{J B}\right)^{2}\left[\frac{\mathrm{d}}{\mathrm{d} \zeta}\left(\boldsymbol{e}_{\theta}+q \boldsymbol{e}_{\zeta}\right)-\frac{1}{2} \frac{\mathrm{d} \ln J}{\mathrm{~d} \zeta}\left(\boldsymbol{e}_{\theta}+q \boldsymbol{e}_{\zeta}\right)\right]
$$

The normal and geodesic components of $\kappa$ are

$$
\kappa_{\mathrm{n}}=\kappa \cdot \frac{\nabla s}{|\nabla s|} \quad \kappa_{\mathrm{g}}=\kappa \cdot\left(\frac{\nabla s}{|\nabla s|} \times \boldsymbol{e}_{\|}\right) .
$$

\section{Drift wave model}

The emphasis of this work is on the effect of geometry through magnetic field gradient and field line curvature. The wave spectrum rather than the driving mechanisms are the main concerns. For this purpose we use the simple cold ion fluid model used by previous authors (Bhattacharjee et al 1983, Persson et al 1996, Persson and Lewandowski 1997), in which electron response is assumed to be close to adiabatic and the cold ion response is derived from the fluid dynamic. The resulting equations are closed through the quasi-neutrality condition to obtain the following electrostatic drift wave eigenvalue equation:

$$
\left[\frac{c_{\mathrm{s}}^{2}}{\omega^{2}} \nabla_{\|}^{2}-\rho_{\mathrm{s}}^{2} \nabla_{\perp}^{2}+\frac{1}{\mathrm{i} \omega}\left(\boldsymbol{v}_{*}+\boldsymbol{v}_{\mathrm{d}}\right) \cdot \nabla_{\perp}+1+\mathrm{i} \delta\right] \frac{e \phi}{T_{\mathrm{e}}}=0
$$

where the time $t$ dependence of the wave potential $\phi$ is assumed to be of the form $\exp (\mathrm{i} \omega t)$, where $\omega$ is the wave frequency. Here $c_{\mathrm{s}}=\sqrt{T_{\mathrm{e}} / m_{\mathrm{i}}}$ is the ion sound speed at the electron temperature, $\rho_{\mathrm{s}}=c_{\mathrm{s}} / \Omega_{\mathrm{ci}}$ and $\Omega_{\mathrm{ci}}$ is the ion cyclotron frequency,

$$
\boldsymbol{v}_{*} \equiv-\frac{T_{\mathrm{e}}}{e B} \boldsymbol{e}_{\|} \times \nabla \ln n_{\mathrm{o}} \quad \boldsymbol{v}_{\mathrm{d}} \equiv \frac{2 T_{\mathrm{e}}}{e B} \boldsymbol{e}_{\|} \times \nabla \ln B
$$

are the diamagnetic and $\nabla B$ drift velocities, respectively, and $n_{\mathrm{o}}$ is the equilibrium plasma density. The last term in equation (4) represents the non-adiabatic part of the electron response which could be due, for example, to collisions, wave-particle resonance, dissipation due to electron trapping, or any other mechanism preventing electrons from moving freely along the field lines. 
Using equation (3) and $\nabla_{\|} \equiv e_{\|} \cdot \nabla$, the operator $\nabla_{\|}^{2}$ can be written as

$$
\nabla_{\|}^{2}=\left.\left(\frac{\dot{\psi} q}{J B}\right)^{2}\left(\frac{\mathrm{d} \ln B}{\mathrm{~d} \zeta} \frac{\mathrm{d}}{\mathrm{d} \zeta}+\frac{\mathrm{d}^{2}}{\mathrm{~d} \zeta^{2}}\right)\right|_{\text {field line }} .
$$

We assume that the wave potential $\tilde{\phi}$ is of the ballooning type, i.e.

$$
\tilde{\phi}=\hat{\phi}(\zeta, \epsilon) \exp \left(-\mathrm{i} \epsilon^{-1} S(\boldsymbol{r})\right)
$$

where $\epsilon$ is the aspect ratio and $S(\boldsymbol{r})$ is a function satisfying $\boldsymbol{e}_{\|} \cdot \nabla S \equiv 0$, so that $S=S(\alpha, q)$. Then, equation (4) with the transformation $\sqrt{B} \hat{\phi}=\Phi$ can be written as:

$\frac{\mathrm{d}^{2} \Phi}{\mathrm{d} \zeta^{2}}+\left[Q-\left(\frac{J B}{q \bar{R} \dot{\psi}}\right)^{2}\left\{\left(\Omega_{*}+\Omega_{\mathrm{d}}\right) \chi \Omega-\left(1+\chi^{2} \hat{\boldsymbol{k}}_{\perp} \cdot \hat{\boldsymbol{k}}_{\perp}+\mathrm{i} \delta\right) \Omega^{2}\right\}\right] \Phi=0$

where

$$
\begin{aligned}
& Q=Q(\zeta)=-\frac{5}{16}\left(\frac{\mathrm{d} \ln J}{\mathrm{~d} \zeta}\right)^{2}+\frac{1}{4 J} \frac{\mathrm{d}^{2} J}{\mathrm{~d} \zeta^{2}} \quad \Omega=\frac{\bar{R} \omega}{c_{\mathrm{s}}} \\
& \Omega_{*}=-\frac{2 \bar{R}}{L_{\mathrm{n}}} \quad \chi=\epsilon^{-1} \frac{q \rho_{\mathrm{so}}}{\bar{a}} \frac{\partial S}{\partial \alpha} \\
& \Omega_{\mathrm{d}}=\Omega_{\mathrm{d}}\left(\zeta, \theta_{k}\right)=\frac{2 \bar{a} \bar{R} B_{\mathrm{o}}}{\bar{a} \mathrm{~d} s}\left(\boldsymbol{e}_{\|} \times \nabla \ln B\right) \cdot\left(\nabla \alpha+\theta_{k} \dot{q} \nabla s\right) \\
& \hat{\boldsymbol{k}}_{\perp}=\hat{\boldsymbol{k}}_{\perp}\left(\zeta, \theta_{k}\right)=\frac{\bar{a} B_{\mathrm{o}}}{q B}\left(\nabla \alpha+\theta_{k} \dot{q} \nabla s\right) \quad \theta_{k}=\frac{\partial S}{\partial q} / \frac{\partial S}{\partial \alpha} .
\end{aligned}
$$

$\bar{R}$ is the average major radius, $\rho_{\text {so }}$ is the ion Larmor radius at the magnetic axis, and $\bar{a}$ and $B_{\mathrm{o}}$ were defined in the previous section. Using equation (3) we can write

$$
\nabla \ln B=\frac{1}{2}\left[\left(\frac{\mathrm{d}}{\mathrm{d} s} \ln \left(B_{\theta}+q B_{\zeta}\right)-\frac{\partial \ln J}{\partial s}\right) \nabla s-\frac{\partial \ln J}{\partial \theta} \nabla \theta-\frac{\partial \ln J}{\partial \zeta} \nabla \zeta\right] .
$$

Equation (5) is an ordinary differential equation to be solved along a magnetic field line numerically subject to appropriate boundary conditions for large $|\zeta|$ and by demanding continuity of the function and its first derivative at a point $\left(\theta_{0}, \zeta_{0}\right)$, usually referred to as a 'matching point'. Hence, at a given magnetic surface $\psi$, the eigenfunction $\Phi$ and the corresponding normalized eigenfrequency $\Omega$ are obtained for given values of the equilibrium plasma density scale length, $L_{\mathrm{n}}$, and the parameters $\chi$ and $\theta_{k}$; the former controls the magnitude of the perpendicular wavevector $\boldsymbol{k}_{\perp}$ and the latter controls the magnitude of its component along the outward normal of the flux surface. The vector $\boldsymbol{k}_{\perp}$ rotates in the perpendicular (to $\boldsymbol{B}$ ) plane when $\theta_{k}$ is increased.

\section{Results}

We have carried out a numerical study of drift waves in a heliac H1-NF magnetic field configuration. The full equilibrium is obtained from VMEC with $n_{\mathrm{p}}=13, n_{\mathrm{t}}=27$, and the field configuration has three-fold toroidal symmetry, so that $N=3$. We solve the drift wave equation (5) numerically in this configuration, applying the WKB (Wentzel-KramersBrillouin) type boundary conditions for large $\zeta$; that is $\Phi$ represents either propagating waves with outgoing energy (non-localized modes) or standing waves (localized modes) beyond a certain point away from the mode centre (matching point) along the field line. Details of the boundary conditions and numerical method used are given in the appendix. The drift wave spectrum thus obtained in the absence of the driving mechanism $(\delta=0.0)$ and for a finite $\delta$ 
at the matching point $\left(\theta_{\mathrm{o}}=0, \zeta_{\mathrm{o}}=0\right)$ on the flux surface $s=0.9 \dot{\psi}$ for the parameter values $\chi=0.8, \theta_{k}=0.0 \mathrm{rad}$ and $L_{\mathrm{n}}=5.0 \mathrm{~cm}$ is shown in figure 1 . The eigenfunctions at different frequencies in the spectrum are shown as insets. In this spectrum, very low frequency modes are highly damped and have extended eigenfunctions along the field line. The negative growth rates of these modes do not depend on the finite $\delta$. With increasing frequency, the modes are first weakly localized and then highly localized along the field line. These modes are marginally stable in the absence of a driving mechanism. For finite $\delta$, the weakly localized modes become damped modes and the strongly localized high frequency modes are driven unstable. The most localized modes have the largest frequency and growth rates. These modes can be classified as symmetric modes, having the same structure across the point of magnetic field symmetry, and odd modes, having the opposite structure across the symmetry point. The spectrum is discrete and may not be fully resolved, especially for intermediate frequencies.

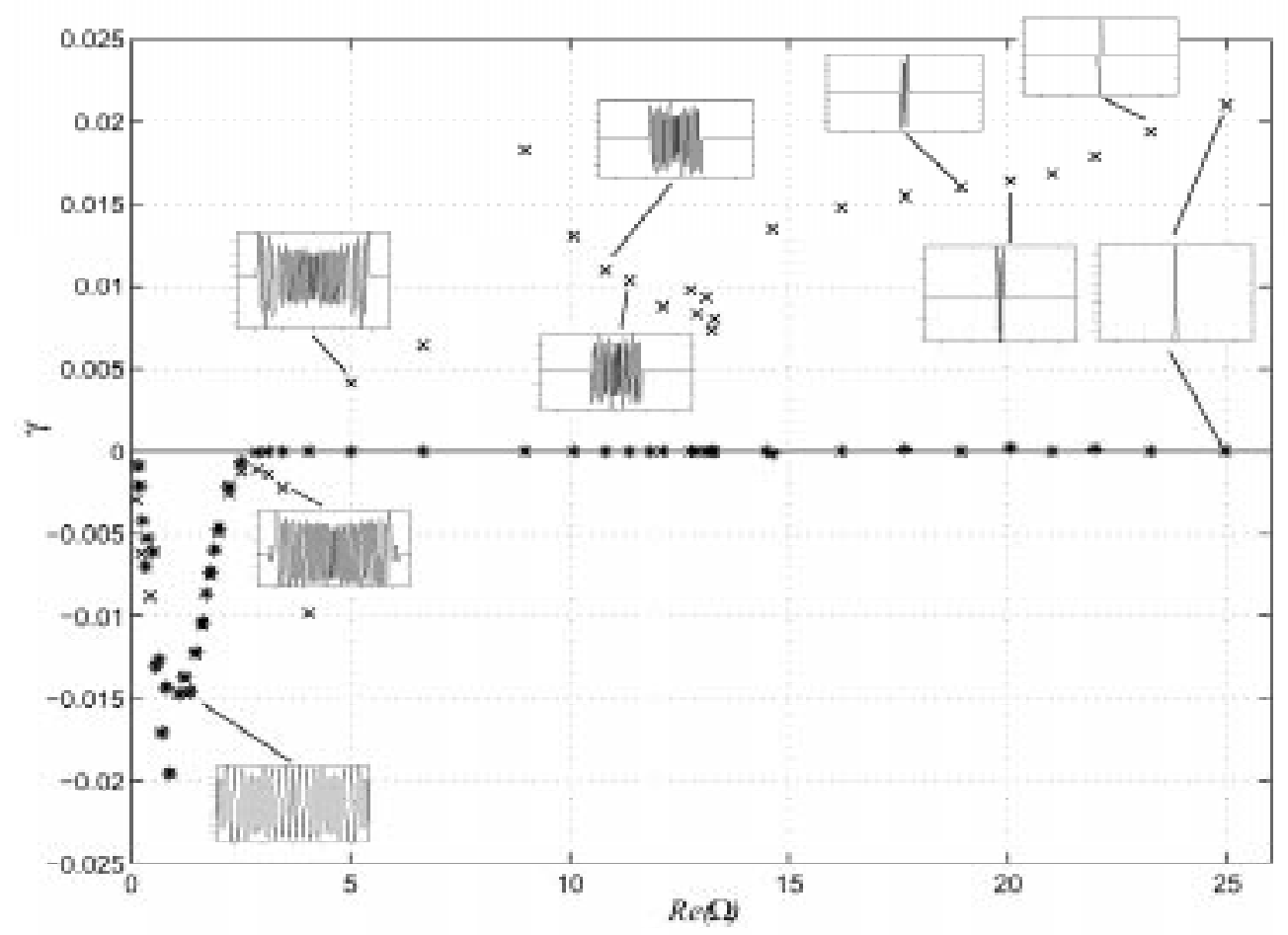

Figure 1. The spectrum of the drift wave equation (the growth rate $\gamma \equiv-\operatorname{Im}(\Omega)$ versus the frequency $\operatorname{Re}(\Omega)$ ) with $\delta=0$ (indicated by ' $*$ ') and $\delta=0.001$ (indicated by ' $\times$ ') obtained at the matching point $\theta_{\mathrm{o}}=\zeta_{\mathrm{o}}=0$.

The potential function (defined as the quantity in square brackets in equation (5)) of the drift wave equation is strongly dependent on the value of the parameter $\chi$, which controls the magnitude of the perpendicular (to $\boldsymbol{B}$ ) wavenumber $\boldsymbol{k}_{\perp}$. This is a free parameter and hence the spectrum variation versus this parameter must be scanned. Figure 2 shows such a scan for the same values of the other parameters as in figure 1 , with $\delta=0.001$. The eigenfunctions of the highest frequency modes for different $\chi$ are shown as insets. For small $\chi$ the frequency spectrum is very narrow. The low frequency modes are the most stable. These modes have extended eigenfunction structures along the field line. The high frequency modes are unstable, having a large growth rate, and are well localized in a wide region along the field line, with broad 
potential envelopes. As $\chi$ increases the spectrum spreads into many dispersion branches. The low frequency stable modes remain almost unaffected, except that their damping rate decreases. The frequencies and growth rates of the unstable localized modes first rapidly increase and then slowly decrease. The potential function becomes more and more localized with narrow envelopes. The growth rate spectrum, shown in figure 2 (right), is somewhat irregular for some $\chi$. This is probably because of the choice of the function $\delta$, which gives a larger driving for extended (weakly localized) modes as it is chosen to be constant along the field line.
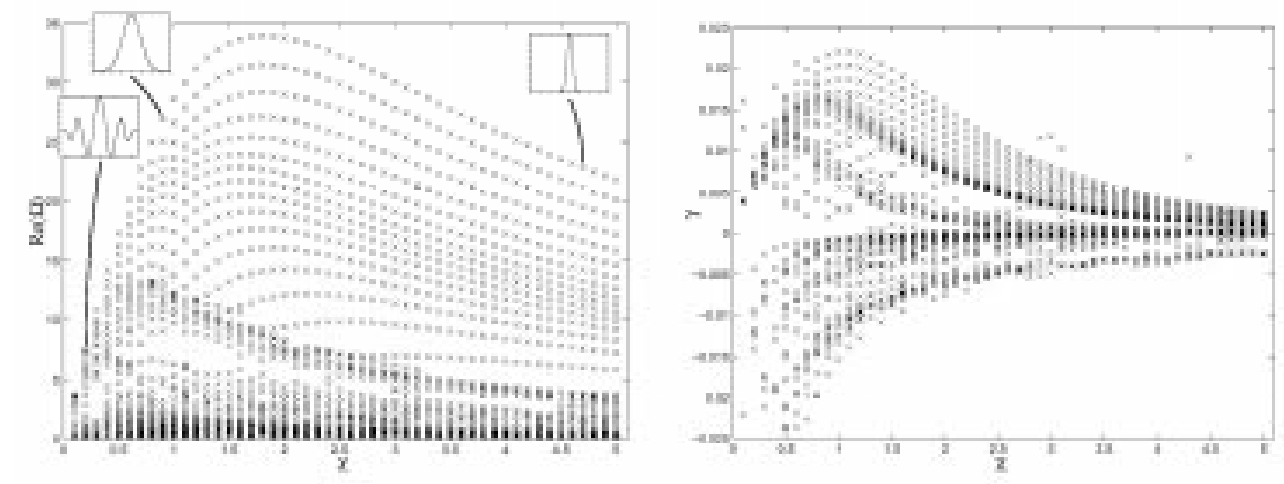

Figure 2. The frequency (left) and the growth rate (right) variation with $\chi$.

In a simple slab geometry, when the parallel ion dynamic and temperature are neglected, the fundamental drift wave dispersion relation is

$$
\Omega=\frac{\Omega_{*} \chi}{\left(1+\chi^{2} \hat{\boldsymbol{k}}_{\perp}^{2}\right)+\mathrm{i} \delta} .
$$

This implies that for $\chi<1$, the frequency $\operatorname{Re}(\Omega)$ and the growth rate $\gamma \equiv-\operatorname{Im}(\Omega)$ are

$$
\operatorname{Re}(\Omega) \approx \Omega_{*} \chi \quad \gamma \approx \Omega_{*} \delta \chi
$$

and for $\chi>1$,

$$
\operatorname{Re}(\Omega) \approx \frac{\Omega_{*}}{\hat{\boldsymbol{k}}_{\perp}^{2} \chi} \quad \gamma \approx \frac{\Omega_{*} \delta}{\hat{\boldsymbol{k}}_{\perp}^{2} \chi} .
$$

So, the frequency and the growth rate are directly proportional to the parameter $\chi$ when $\chi<1$, and are inversely proportional when $\chi>1$. Our numerical results for each mode in the drift wave spectrum are consistent with the fundamental drift wave theory, though the growth rates of the damped modes for small $\chi$ and those of unstable modes for $\chi$ near zero have a different behaviour, which is due to neglecting the parallel ion dynamic.

The parameter $\theta_{k}$ controls the component of the wavevector along the normal to the flux surface and appears in the wave equation through $\hat{\boldsymbol{k}}_{\perp}$. This is also a free parameter and so the spectrum should be scanned versus this parameter. Results of such a scan, keeping other involved parameters constant at the values given above, are shown in figure 3. Eigenfunctions of the highest frequency modes are shown in the insets. Near $\theta_{k}=0$ the frequency spectrum of unstable localized modes is broad. As $\theta_{k}$ increases, the frequencies and growth rates of these modes decrease and the corresponding eigenfunctions are shifted away from the symmetry point of the magnetic field and have a broader envelope. For large values of $\theta_{k}$ the strongly localized modes cease to exist. However, the low frequency stable modes with negative growth rates exist for all values of $\theta_{k}$ and their frequency spectra remain almost the same, though their 

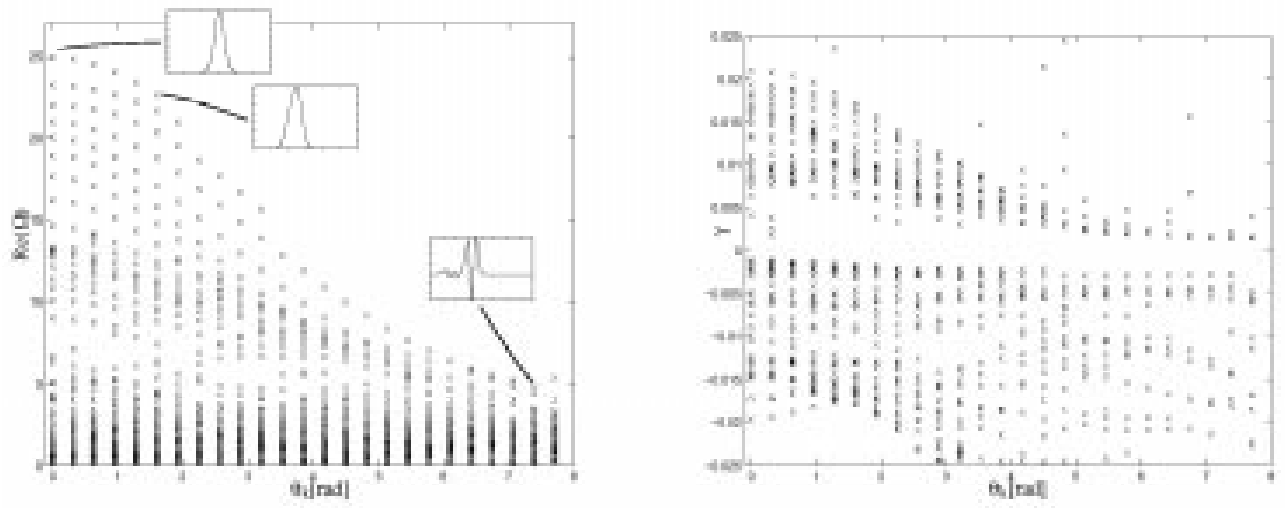

Figure 3. The frequency (left) and the growth rate (right) variation with $\theta_{k}$.

damping rates tend to increase with $\theta_{k}$. There is some irregular behaviour in the growth rate spectrum, which could be due to the choice of the instability driving function $\delta=$ const

Of particular experimental interest is the most localized unstable mode on the $\chi-\theta_{k}$ plane. Such a scan, obtained at the matching point $\left(\theta_{\mathrm{o}}=0, \zeta_{\mathrm{o}}=0\right)$ for the parameter $\mathrm{E}_{\mathrm{n}}=5 \mathrm{~cm}$ and $\delta=0.001$, is shown in figure 4 . The frequency and growth rate of the mode first increase and then decay as $\chi$ increases, and decay as $\theta_{k}$ increases. The maximum frequency and growth rate are obtained around $\chi=1.0, \theta_{k}=0.0$.
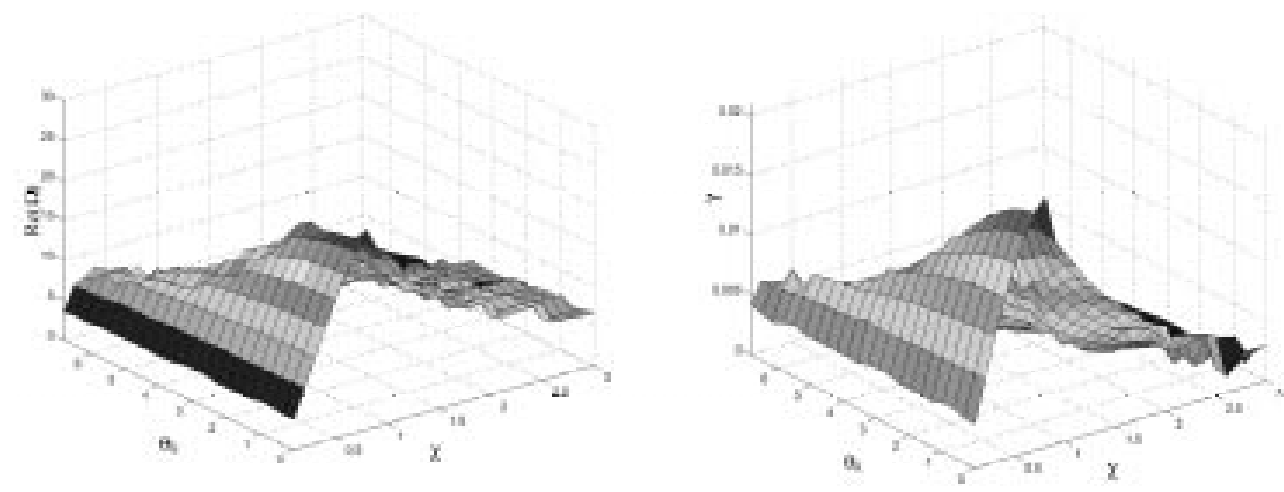

Figure 4. The frequency (left) and the growth rate (right) of the most localized mode versus $\chi-\theta_{k}$ space.

The density scale length $\biguplus_{n}$, which appears in the wave equation through diamagnetic drift velocity, is given by the plasma density profile. Figure 5 shows a scan of drift wave frequency and growth rate spectra versus $\biguplus_{n}$, where the insets show the wave potential for the first and second most localized modes. For a steep density profile, $\mathrm{E}_{\mathrm{n}}$ is small and the spectrum is very wide. The frequencies and growth rates of the localized unstable modes and of damped modes are large. The eigenfunctions of the localized modes have very narrow envelopes. As the density profile is made flat and $\mathrm{E}_{\mathrm{n}}$ is increased, the frequencies and growth rates of these modes decrease exponentially, the spectrum gets more narrow and the eigenfunction envelopes of the localized modes broaden. When the density profile is flat and $\mathrm{t}_{\mathrm{n}}$ is large, only highly localized modes are excited with small growth rates. 

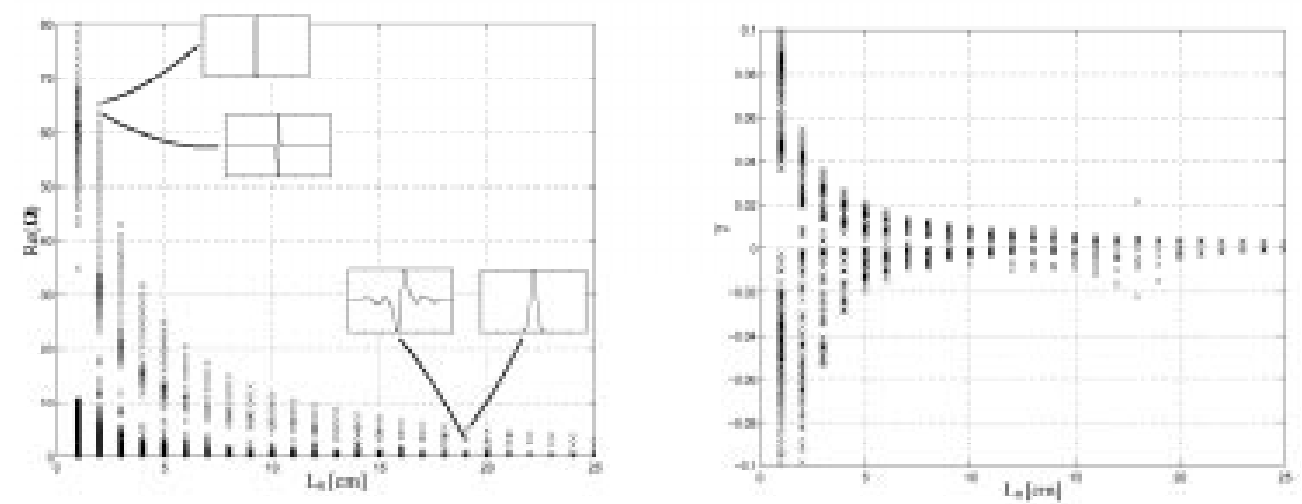

Figure 5. The frequency (left) and the growth rate (right) variation with the density scale length $L_{\mathrm{n}}$.

The spectrum can also vary with the position of the matching point on the flux surface because of the variation in the equilibrium quantities. We have calculated this variation by moving the matching point $\left(\theta_{\mathrm{o}}, \zeta_{\mathrm{o}}\right)$ along the field line, passing through the point $(\theta=0, \zeta=0)$ for the same values of the parameters as in figure 1 , with $\delta=0.001$ and $\theta_{k}=\theta_{0}$. The results are shown in figure 6 , where the insets show the potential function of the most localized mode at different positions along the field line. The high frequency localized unstable modes are excited in the regions of maximum normal curvature where the field line is completely concave outward or inward. These modes are localized around the points of symmetry of the field configuration, which occur in the covering space at $\theta=n \pi, \zeta=n \pi / 3$, where $n=0,1,2, \ldots$. The eigenfunctions have either symmetric or antisymmetric structures across these points. The low frequency, weakly localized modes are found everywhere and their frequency spectra vary along the field line in the form of many interacting branches. The spectrum of the very low frequency damped modes remains almost the same along the field line, though their damping rate spectrum varies. The variation in the normal and geodesic components of the curvature vector and the magnetic field strength along the field are also shown in figure 6 . The spectrum has no clear relation with curvature and magnetic field, except that in the region where normal curvature and magnetic field are large, only strongly localized modes are excited at high frequencies and their spectra are very discrete.

The localization of the drift waves on the magnetic surface is of great interest. We have calculated the drift wave spectrum for the same parameter values as in figure 1 , with $\theta_{k}=\theta_{\mathrm{o}}$. The variation in the spectrum along the $\theta=0.0$ and $\zeta=0.0$ lines on the magnetic surface are shown in figure 7 , where the insets show the eigenfunctions of the most localized modes in different regions along the line. The variation in the curvature components and magnetic field strength are also shown. The strongly localized high frequency modes are excited only around the points of symmetry of the field configuration. The low frequency, weakly localized modes are excited everywhere. Their frequency spectra vary in the form of interacting branches along the $\theta=0.0$ line and do not vary much along the $\zeta=0.0$ line. The spectrum of the low frequency damped modes remains almost unchanged, while the damping rate spectrum changes significantly in the region where the normal curvature and magnetic field are large. Apparently, the frequency spectra do not show any remarkable relation with the curvature and magnetic field variation, except that in the region of large normal curvature and magnetic field, only strongly localized modes are excited at high frequencies and their spectrum is very discrete. 

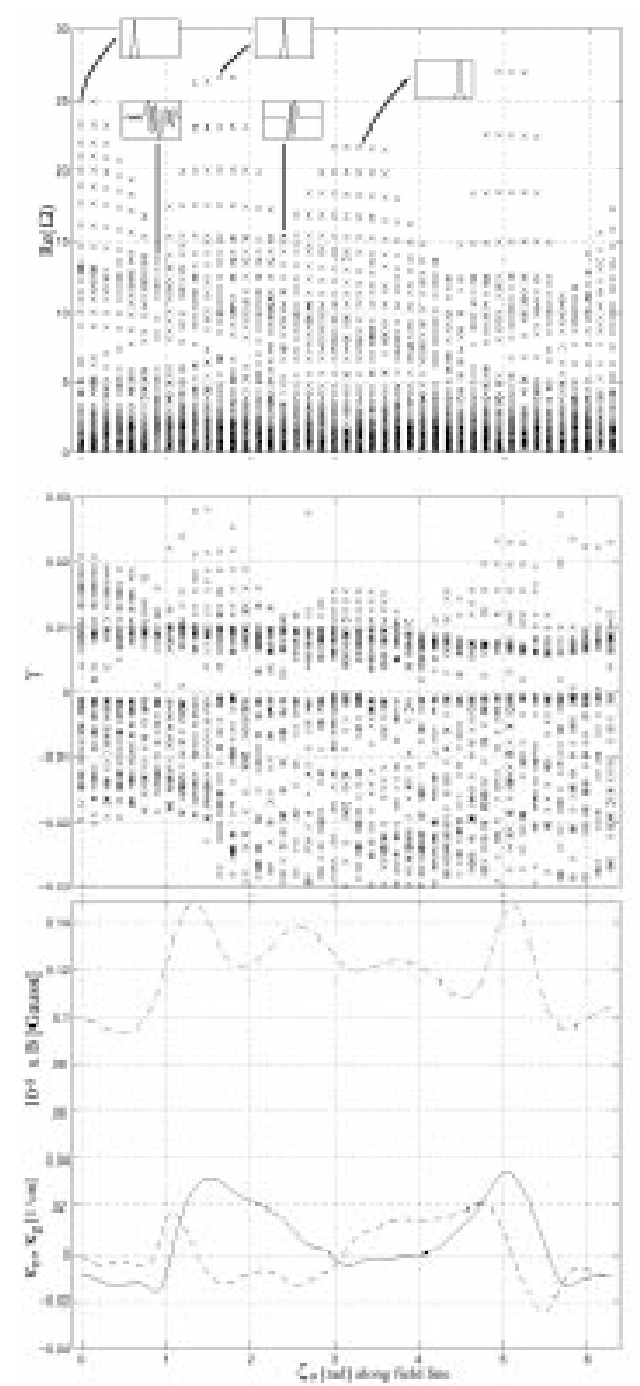

Figure 6. Variation in the frequency (top) and growth rate (middle) spectra and change in $B, \kappa_{\mathrm{n}}$ and $\kappa_{\mathrm{g}}$ (bottom) along the field line passing through $\theta=\zeta=0 . B$ is the dashed-dotted curve, $\kappa_{\mathrm{g}}$ is the dashed curve, and $\kappa_{\mathrm{n}}$ is the solid curve.

The frequency and growth rate for the most localized mode on the magnetic surface are shown in figure 8 . They change periodically over the surface, attaining large values at the symmetry points where the field lines are completely concave outward or inward.

Theoretically, as discussed by Dewar and Glasser (1983), since all the equilibrium quantities are periodic functions of the coordinates $\theta$ and $\zeta$, with periods $2 \pi$ and $2 \pi / 3$, respectively, in the H1-NF device, all the eigenvalues $\Omega$ must have the same periodicity over the matching points $\left(\theta_{\mathrm{o}}, \zeta_{\mathrm{o}}\right)$-space. This is provided that when $\theta_{\mathrm{o}}$ is varied, $\theta_{k}$ is also varied by the same amount so that the orientation of the perpendicular wavevector $\boldsymbol{k}_{\perp}$ remains the same. Our numerical results shown in figures 7 and 8 are consistent with this, that is, the frequency $\operatorname{Re}(\Omega)$ and the growth rate $\gamma$ of each mode in the spectrum have the periodicity predicted by theory. 

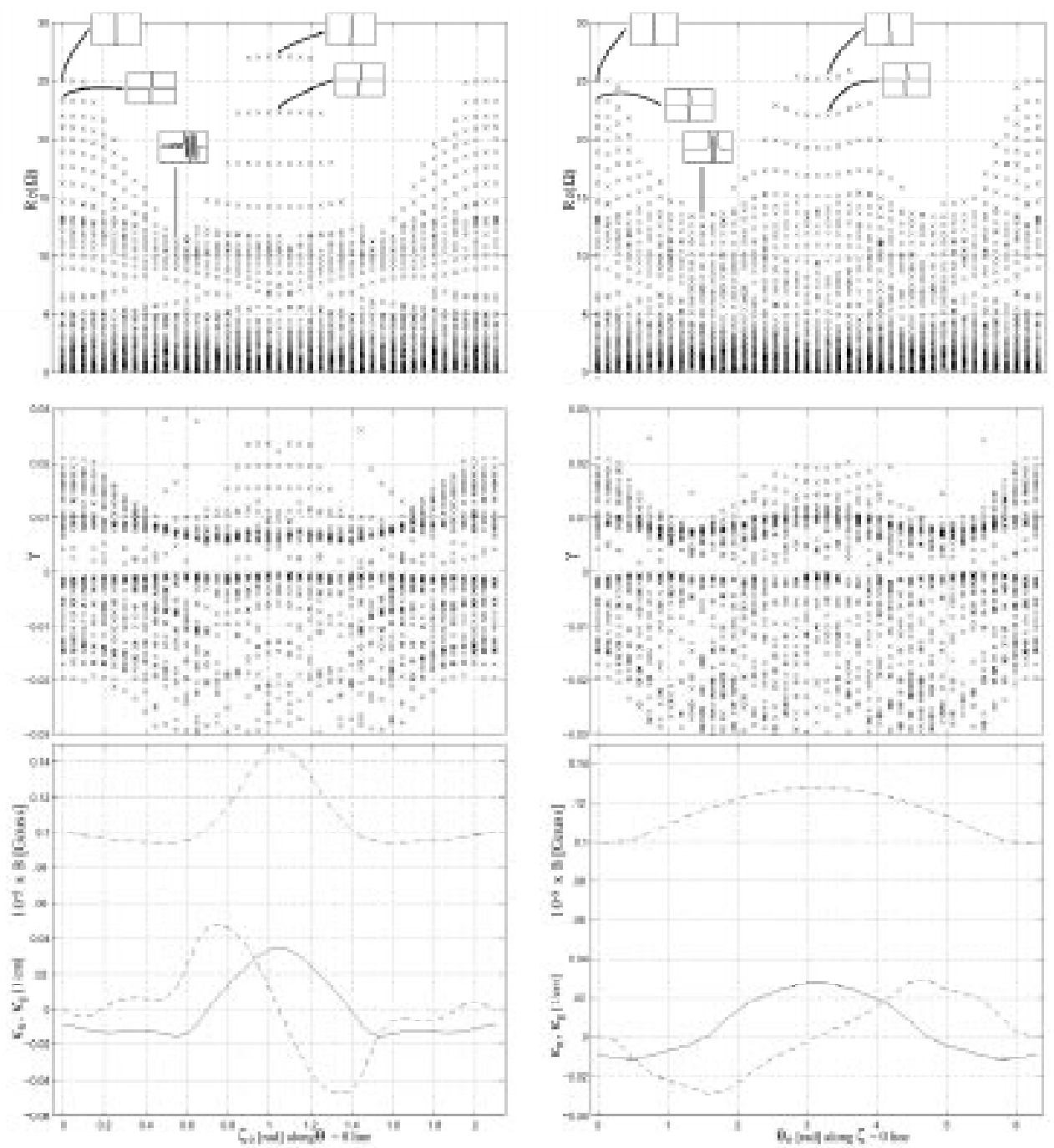

Figure 7. Variation in the frequency (top) and growth rate (middle) spectra and change in $B, \kappa_{\mathrm{n}}$ and $\kappa_{\mathrm{g}}$ (bottom) along the $\zeta=0$ (left) and $\theta=0$ (right) lines. $B$ is the dashed-dotted curve, $\kappa_{\mathrm{g}}$ is the dashed curve, and $\kappa_{\mathrm{n}}$ is the solid curve.

The variation in the magnetic field and the normal, and the components of the curvature vector on the real three-dimensional magnetic flux surface are shown in figures 9 and 10. The magnetic field $B$ and the normal curvature $\kappa_{\mathrm{n}}$ are maximum in the region where field lines are concave inward and are minimum where field lines are concave outward. The geodesic curvature $\kappa_{\mathrm{g}}$ have extreme values around the points where the maximum twist of the field lines is in the poloidal direction. 

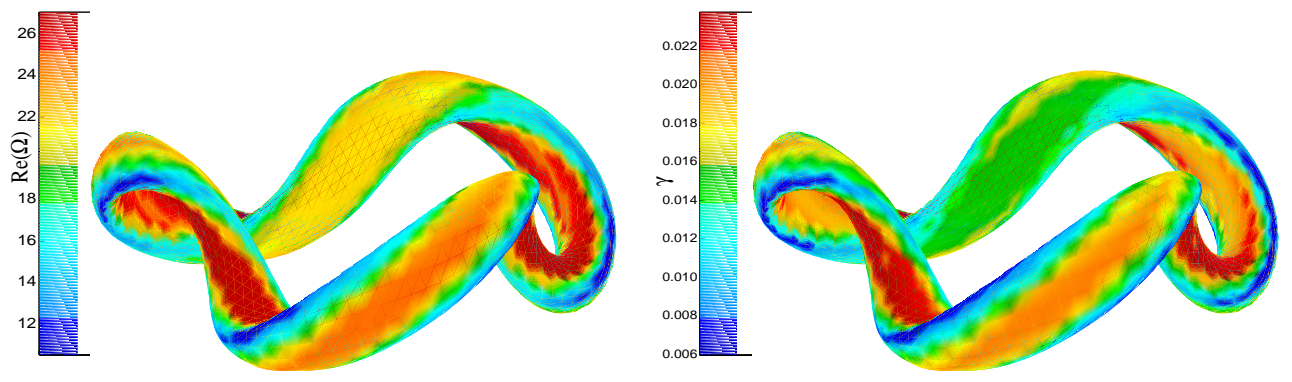

Figure 8. The frequency $\operatorname{Re}(\Omega)$ (left) and the growth rate $\gamma \equiv-\operatorname{Im}(\Omega)$ (right) of the most localized mode on the flux surface at $s=0.9 \dot{\psi}$.

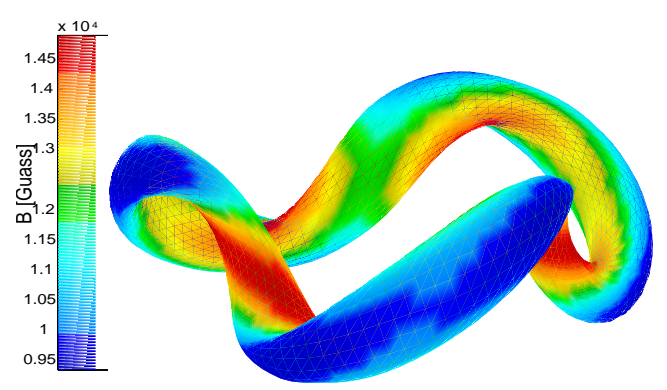

Figure 9. The magnetic field $B$ on the flux surface at $s=0.9 \dot{\psi}$.
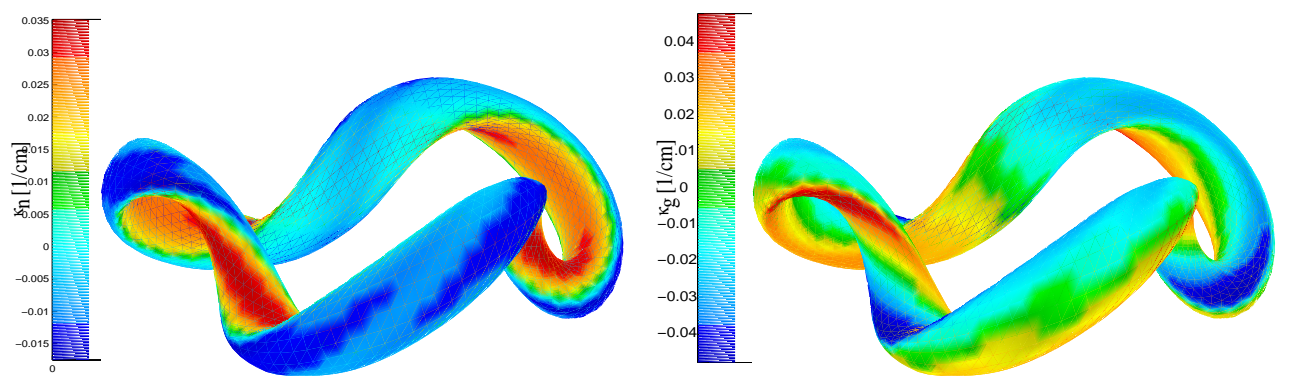

Figure 10. The normal, $\kappa_{\mathrm{n}}$ (left), and geodesic, $\kappa_{\mathrm{g}}$ (right), components of the curvature vector $\kappa$ on the flux surface at $s=0.9 \dot{\psi}$.

\section{Discussion and conclusion}

In the straight stellarator model geometry study by Persson and Lewandowski (1996) the lower frequency modes were more localized and the non-localized modes were found at higher frequencies. In the present three-dimensional study this trend is quite reversed. The more localized modes were found at higher frequencies and the non-localized modes had lower frequencies. This difference is due to the toroidal curvature of the three-dimensional magnetic field configuration. This type of spectral behaviour is obtained when the potential function of the wave equation is a monotonically decreasing function of the frequency. The spectral behaviour found in the straight case is obtained when it is a monotonically increasing function of the frequency, which can easily be seen by solving a simple eigenvalue problem analytically. 
In the straight case, this behaviour of the potential function is obtained because the function $\left|\hat{\boldsymbol{k}}_{\perp}\right|$, on average strongly increases along the field line. In real stellarator geometry, however, this function is almost constant, on average, which makes the potential function a decreasing function of the frequency over the interval the spectrum is found.

To conclude, we have discussed a method of calculating the real three-dimensional stellarator equilibrium magnetic field configuration. Using this method a computer code has been developed which can compute any stellarator equilibria. With this code, drift waves are investigated in a stellarator called heliac H1-NF using ballooning mode formalism. The drift wave frequency spectrum and the corresponding growth rate spectrum are found for typical values of the parameters. In this spectrum low frequency modes are very stable and their negative growth rate does not depend on the instability mechanism. The high frequency modes are marginally stable and localized along the field line, which can be driven unstable by a dissipative mechanism. For a steep plasma density profile, the spectrum is very wide and the frequencies and growth rates of the localized unstable modes are large, and for flat density profiles, the spectrum is very narrow with only highly localized modes being excited, the growth rates of which are small. The spectrum of the long wavelength modes is very narrow and almost continuous. As the wavelength is reduced, the spectrum splits into many discrete branches at large frequencies; first the frequencies and growth rates increase rapidly and then slowly decrease. The drift wave spectrum is also found to be strongly dependent on the orientation of the wavevector and the position in plasma. The strongly localized high frequency modes are dispersed in a small angle, around a direction in the magnetic surface perpendicular to the magnetic field. These modes are excited only in the regions around the symmetry points of the magnetic field configuration, where the field lines are completely concave outward or inward. The spectrum in the region where the normal curvature and magnetic field are large is very discrete, with only highly localized modes being excited at high frequencies.

The driving mechanism appears in our drift wave equation through the function $\delta$, which we have kept as a fixed constant in the present study. In a more realistic model, $\delta$ is a complex function depending on both geometry and physics. Another limitation of the present study is that we have used a cold ion model to derive our wave equation and ignored particle trapping in the local magnetic wells, as well as toroidal trapping which may only be justified in the electron-neutral collision dominated regime where the electron temperature is much larger than the ion temperature. Furthermore, by assuming that the wave potentials are of a ballooning type we have solved the wave equation locally, neglecting the radial structure of the wave potential. To investigate the global drift wave spectrum either the wave equation should be solved simultaneously in radial and parallel (to $\boldsymbol{B}$ ) directions, or the local structure should be coupled to a ray tracing algorithm, as done by Dewar and Glasser (1983) or to a surface construction algorithm like that used by Cooper et al (1996).

\section{Appendix. Boundary conditions and numerical method}

The boundary conditions for $\Phi$ far away from the matching point are that $\Phi$ either represents outgoing waves or standing waves along the field line. That is, at a large $\zeta=\zeta_{\max }$

$$
\Phi=\Phi_{\mathrm{o}} \exp (\varphi(\zeta, \Omega))
$$

where $\Phi_{\mathrm{o}}$ is a constant and $\varphi$ is a complex function satisfying either of the following conditions:

$$
\left.\begin{array}{lll}
\operatorname{Im}\left(\varphi\left(\zeta_{\max }, \Omega\right)\right)>0 & \operatorname{Im}\left(\varphi\left(-\zeta_{\max }, \Omega\right)\right)<0 & \text { for outgoing waves } \\
\operatorname{Re}\left(\varphi\left( \pm \zeta_{\max }, \Omega\right)\right) \approx 0 & \operatorname{Re}\left(\varphi\left( \pm \zeta_{\max }, \Omega\right)\right)<0 & \text { for standing waves. }
\end{array}\right\}
$$


Writing the eigenvalue equation (5) in the form

$$
\frac{\mathrm{d}^{2} \Phi}{\mathrm{d} \zeta^{2}}+F(\zeta, \Omega) \Phi=0
$$

and substituting equation (6) into this, we get

$$
\left(\varphi^{\prime}\right)^{2}+\varphi^{\prime \prime}+F=0
$$

Here the prime represents a derivative w.r.t. $\zeta$. Assuming that $\varphi^{\prime}$ is a slowly varying function of $\zeta$, so that $\varphi^{\prime \prime} \ll \varphi^{\prime}$, the solutions of this nonlinear equation up to the first order of smallness parameter $\varphi^{\prime \prime} / \varphi^{\prime}$ are given by the equation

$$
\varphi^{\prime}= \pm \mathrm{i} \sqrt{F}-\frac{1}{4}(\ln (F))^{\prime} .
$$

Substituting this into equation (6), one obtains

$$
\Phi^{\prime}=\left[ \pm \mathrm{i} \sqrt{F}-\frac{1}{4}(\ln (F))^{\prime}\right] \Phi .
$$

These are the standard WKB boundary conditions. The solution fulfilling condition (7) is given by

$$
\left.\begin{array}{rlr}
\Phi^{\prime}= & {\left[-\mathrm{i}|\operatorname{Re}(\sqrt{F})|-\operatorname{sign}(\operatorname{Re}(\sqrt{F})) \operatorname{Im}(\sqrt{F})-\frac{1}{4}(\ln (F))^{\prime}\right] \Phi} & \text { at } \zeta=\zeta_{\max } \\
= & {\left[\mathrm{i}|\operatorname{Re}(\sqrt{F})|-\operatorname{sign}(\operatorname{Re}(\sqrt{F})) \operatorname{Im}(\sqrt{F})-\frac{1}{4}(\ln (F))^{\prime}\right] \Phi} & \text { at } \zeta=-\zeta_{\max } \\
& \text { when } \operatorname{Re}(\sqrt{F}) \approx 0 & \\
\Phi^{\prime}=\left[-|\operatorname{Im}(\sqrt{F})|-\frac{1}{4}(\ln (F))^{\prime}\right] \Phi & \text { at } \zeta= \pm \zeta_{\max } .
\end{array}\right\}
$$

The function $F(\zeta, \Omega)$ is pre-calculated on the domain $\left[-\zeta_{\max }+\zeta_{0}, \zeta_{\max }+\zeta_{0}\right]$ for a given $\Omega$. The eigenfunction $\Phi$ at $\zeta= \pm \zeta_{\max }$ is fixed arbitrarily, the corresponding value of $\Phi^{\prime}$ is calculated using equation (9), and $\Phi$ at $\zeta= \pm\left(\zeta_{\max }-\Delta z t\right)$ ( $\Delta z t$ is the integration step length) is calculated using the Taylors expansion. Then $\Phi$ is determined all over the domain using a sixth-order Numerov scheme (Gladd and Horton 1973):

$$
\begin{aligned}
\Phi(\zeta-\Delta \zeta)[1 & \left.+\frac{(\Delta \zeta)^{2}}{12} F(\zeta-\Delta \zeta)\right]=\left[2-\frac{5}{6}(\Delta \zeta)^{2} F(\zeta)\right] \Phi(\zeta) \\
- & {\left[1+\frac{(\Delta \zeta)^{2}}{12} F(\zeta+\Delta \zeta)\right] \Phi(\zeta+\Delta \zeta) . }
\end{aligned}
$$

The function $\Phi$ is calculated at the matching point $\zeta_{\mathrm{o}}$ from the right, say $\Phi_{+}$, and from the left, say $\Phi_{-}$, for a given frequency $\Omega$. The values of $\Omega$ at which $\Phi_{+}$and $\Phi_{-}$match are the eigenfrequencies. These are calculated by finding roots (zeros) of the function

$$
f(\Omega)=\frac{\Phi_{+}^{\prime}}{\Phi_{+}}-\frac{\Phi_{-}^{\prime}}{\Phi_{-}}
$$

on the complex frequency plane. The plane is divided into a number of small areas, each of which is then independently searched to find the roots using a Müller algorithm (Press et al 1992), which generalizes the secant method by using quadratic interpolations.

The computer program solving this eigenvalue problem takes about 2 min to calculate the equilibrium part of the function $F(\zeta, \Omega)$ and less than 3 min to calculate the whole spectrum presented in figure 1 on a SGI Cray Origin 2000 super computer.

\section{References}

Bhattacharjee A, Sedlak J E, Similon P L, Rosenbluth M N and Ross D W 1983 Phys. Fluids 26880

Boozer A H, Baldwin D E, Horton C W, Dominguez R, Glasser A H, Krommes J A, Neilson G H, Shaing K, Sadowski W L and Weitzner H 1990 Phys. Fluids B 22870 
Cooper W A, Singleton D B and Dewar R L 1996 Phys. Plasmas 3275

Dewar R L and Glasser A H 1983 Phys. Fluids 263038

D'haeseleer W D, Hitchon W N G, Callen J D and Shohet J L 1991 Flux Coordinates and Magnetic Field Structure (Berlin: Springer) ch 6

Dominguez N, Carreras B A, Lynch V E and Diamond P H 1992 Phys. Fluids B 42894

Gladd N T and Horton W 1973 Phys. Fluids 16879

Hamberger S M, Blackwell B D, Sharp L E and Shenton D B 1990 Fusion Technol. 17123

Hastie R J, Hesketh K W and Taylor J B 1979 Nucl. Fusion 191223

Hirshman S P and Betancourt O 1991 J. Comput. Phys. 9699

Pearlstein L D and Berk H L 1969 Phys. Rev. Lett. 23220

Persson M and Lewandowski J L V 1997 Plasma Phys. Control. Fusion 391941

Persson M, Lewandowski J L V and Nordman H 1996 Phys. Plasmas 33720

Press W S, Teukolsky S A, Vetterling W T and Flannery B P 1992 Numerical Recipes in Fortran (New York: Cambridge University Press)

Redd A J, Kritz A H, Bateman G, Rewoldt G and Tang W M 1999 Phys. Plasmas 61162

Ross D W and Mahajan S M 1978 Phys. Rev. Lett. 40324

Rutherford P H and Frieman E A 1968 Phys. Fluids 11569

Strand P, Nordman H, Weiland J and Christiansen J P 1998 Nucl. Fusion 38545

Stroth U 1998 Plasma Phys. Control. Fusion 409

Tang W M, Rewoldt G and Chen L 1986 Phys. Fluids 293715 